\title{
Review Article \\ Simplifying Thromboprophylaxis Could Improve Outcomes in Orthopaedic Surgery
}

\author{
Richard J. Friedman \\ Department of Orthopaedic Surgery, Roper Hospital, Charleston Orthopaedic Associates, 1012 Physicians Drive, \\ Charleston, SC 29414, USA \\ Correspondence should be addressed to Richard J. Friedman, rjfriedman@mybones.com
}

Received 6 February 2010; Revised 27 May 2010; Accepted 18 August 2010

Academic Editor: Bergqvist Bergqvist

Copyright ( $\odot 2010$ Richard J. Friedman. This is an open access article distributed under the Creative Commons Attribution License, which permits unrestricted use, distribution, and reproduction in any medium, provided the original work is properly cited.

\begin{abstract}
Venous thromboembolism is a serious complication after total hip or knee surgery, and there is a well-established clinical need for thromboprophylaxis. However, in a large number of cases adequate administration of thromboprophylaxis does not seem to occur after total joint arthroplasty. A major challenge in the management of thromboprophylaxis is to balance the benefits of treatment with the risks, including bleeding complications. Another potential barrier to the optimal use of thromboprophylaxis could be the inconvenience of currently available agents. Many surgeons therefore adopt a conservative approach towards thromboprophylaxis. Simplifying therapy with more convenient, efficacious, and safe anticoagulants could change attitudes to anticoagulant use and improve adherence to thromboprophylactic guidelines.
\end{abstract}

\section{Introduction}

Venous thromboembolism (VTE) is a serious complication after major orthopaedic surgery [1]. The rates of venographic deep vein thrombosis (DVT) and proximal DVT 7 to 14 days after major orthopaedic surgery in patients who receive no thromboprophylaxis are approximately $40 \%$ to $60 \%$ and $10 \%$ to $30 \%$, respectively [1]. The manifestation of DVT is, to some extent, a consequence of bone damage during surgery, when procoagulant debris triggers thrombin generation, resulting in hypercoagulability [2]. In addition to hypercoagulability, the other components of Virchow's triad of venous stasis and endothelial damage are also thought to play a part in thrombosis [3]. Thus, there is a well-established clinical need for thromboprophylaxis after arthroplasty [1].

A major challenge in the management of anticoagulants is to balance the benefits of treatment with the risks, including bleeding complications. Many surgeons appear concerned about postoperative bleeding and tend to adopt a conservative approach towards the relative risks and benefits of thromboprophylaxis [2]. Consequently, although evidence-based guidelines and recommendations advocate the use of anticoagulants after major orthopaedic surgery, thromboprophylaxis is still used suboptimally [4-6]. However, the evidence that careful prophylaxis administered at an appropriate time after surgery causes surgical bleeding is sparse [7]. In this paper, current trends in thromboprophylaxis after orthopaedic surgery in the United States (US) are described. Factors limiting appropriate implementation of thromboprophylaxis regimens are also discussed.

\section{Current Standard of Care}

Further to the consensus document developed by the National Institute of Health in 1986 [8], there have been a series of American College of Chest Physicians (ACCP) guidelines published on the use of pharmacological agents for thromboprophylaxis after total hip arthroplasty (THA) and total knee arthroplasty (TKA), last updated in 2008 [1].

In the US, the available options for anticoagulation and thromboprophylaxis after elective THA or TKA are the vitamin $\mathrm{K}$ antagonists (VKAs, e.g., warfarin), the lowmolecular-weight heparins (LMWHs), and fondaparinux (an indirect Factor Xa inhibitor). Each of these options is associated with significant limitations that complicate use 
in clinical practice. VKAs have been the mainstay of oral anticoagulant therapy for more than 60 years [9]. However, VKAs have unpredictable pharmacokinetics and pharmacodynamics and significant inter- and intrapatient variability in dose-response relationships. They are associated with multiple drug-drug and food-drug interactions and have a narrow therapeutic window [9]. Regular coagulation monitoring is therefore required to ensure that the international normalized ratio is within the recommended range of 2.0 to 3.0. The heparins are administered subcutaneously, which means that patients often require daily appointments or a nurse visit to administer their medication. LMWHs are also associated with the risk of developing heparin-induced thrombocytopenia [10]. Fondaparinux is also administered subcutaneously and is contraindicated in patients with severe renal impairment and in those that weigh less that $50 \mathrm{~kg}$. In patients over the age of 75 who have undergone THA or TKA, fondaparinux causes an increased risk of bleeding [11].

The timing of initiation of prophylaxis depends upon the type of anticoagulant used. Warfarin therapy is generally initiated prior to surgery because of its delayed onset of action whereas prophylaxis with LMWH can be started 10-12 hours before or 12-24 hours after surgery. There does not seem to be a clear advantage with either regimen, and both regimens are recommended by the ACCP [1]. Thromboprophylaxis is recommended to continue for at least 10 days after joint replacement surgery, with extended prophylaxis for up to 35 days recommended for those patients undergoing THA surgery and with a suggestion that thromboprophylaxis for up to 35 days could be beneficial for those undergoing TKA [1]. Traditionally, thromboprophylaxis used to continue only until the patient was discharged from hospital [12] despite the fact that this could be a suboptimal duration [13] and the risk of DVT and mortality after discharge is considerable [14, 15]. The median length of stay in US hospitals is now as short as 3 days after THA and 4 days after TKA [16]. A retrospective study of the medical records of 3,778 orthopaedic surgery patients found that $88 \%$ were discharged from hospital and prescribed warfarin or acetylsalicylic acid [6].

\section{Suboptimal Utilization of Thromboprophylaxis}

Despite the fact that thromboprophylaxis is now recommended for routine use after total joint arthroplasty, it is not always used optimally. Approximately $10 \%$ of patients received inadequate in-hospital thromboprophylaxis, and approximately $33 \%$ received inadequate postdischarge thromboprophylaxis according to findings from the US Hip and Knee Registry (1996-2001) [17]. An analysis of the data from the multinational Global Orthopaedic Registry (GLORY) to evaluate the compliance of surgeons with the ACCP guidelines for the prevention of VTE showed that only $47 \%$ of THA patients and $61 \%$ of TKA patients received prophylaxis in accordance with the recommended start time, duration, and dose/treatment intensity recommended by the guidelines [16]. Although nearly all patients received prophylaxis on the first day after surgery, more than a quarter did not receive any form of prophylaxis 7 days after surgery [18].

Suboptimal thromboprophylaxis decreases patient outcomes, resulting in many patients remaining at unnecessary risk of thrombosis and its complications [4]. The reasons for lack of compliance with the guidelines may be numerous. They include lack of awareness, poor understanding or disagreement with guidelines (either specifically or as a general concept), resistance to changing established practices, and doubt that a new approach will change outcomes. Established surgeons may also be reluctant to use new anticoagulant regimens because of a fear of increased bleeding risk [17]. Attitudes may also limit a physician's willingness to follow guidelines. An awareness of the guidelines does not necessarily mean that physicians have sufficient knowledge to critically evaluate and apply recommendations [4].

Other potential barriers include the mistaken belief that a small asymptomatic DVT is not important because it cannot cause clinically significant pulmonary embolism (PE) [19], which fortunately is only held by a minority [20]. Due to the often clinically silent nature of VTE, and the low incidence of VTE during the short postoperative hospital stay, the chances of a surgeon witnessing a major DVT or an acute PE are rare [4]. In addition, the trend towards earlier hospital discharge means that many symptomatic events occur after that time $[21,22]$, and patients are often seen by other specialists when referred back to hospital with a venous thromboembolic event; therefore, surgeons are often unaware of the true incidence of VTE in their patients.

Long-term sequelae of VTE are frequent and often disabling [23]. Recurrent VTE can occur after surgery although the incidence is less than in other patients groups such as those with cancer [24]. Thrombosis damages the deep venous valves resulting in venous reflux and venous hypertension of the lower limbs. This residual venous obstruction and inflammation are thought to be responsible for the development of postthrombotic syndrome $[25,26]$. Chronic thrombotic pulmonary hypertension, which is associated with considerable morbidity and mortality, occurs in approximately 3\%-4\% of patients over 2 years after a symptomatic PE [27].

\section{Economic Impact of Venous Thromboembolism}

The acute and chronic phases of VTE-related care have substantial economic consequences $[28,29]$ that can be effectively modeled [30]. A recent study found that the total annual healthcare cost for a VTE ranged from $\$ 7,594$ to $\$ 16,644$, depending on the type of event and whether it was a primary or secondary diagnosis. The hospital readmission rates for DVT or PE within 12 months were 5.3\% for primary and $14.3 \%$ for secondary diagnoses [31]. These data indicate that thromboprophylaxis with anticoagulants should not only be beneficial to patients but could also be cost effective for the healthcare system $[32,33]$. 


\section{Need for More Convenient Anticoagulants}

Another potential barrier to the optimal use of thromboprophylaxis could be the inconvenience of currently available agents [34]. Orthopaedic surgeons and their patients would benefit from an oral anticoagulant that could be administered in fixed doses [35].

\section{Simplifying Therapy}

Noncompliance can result in a poor quality of life and increased medical expenditures in managed care. In a study of diabetic patients, total medical costs were approximately $\$ 4,500$ for patients at $80 \%-100 \%$ adherence compared with approximately $\$ 8,900$ for those at $1 \%-19 \%$ adherence [36]. A variety of factors affect noncompliance, but simplifying treatment has been shown to improve adherence in asthma patients [37], and cardiovascular patients given single-pill amlodipine/atorvastatin were found to be approximately three times more likely to achieve adherence over 1 year of followup than patients given a two-pill regimen [38]. Similarly, simplifying therapy to a once-daily regimen in virologically suppressed HIV-1-infected patients improved adherence and patient satisfaction [39].

\section{Novel Anticoagulants}

Anticoagulants in development are targeting different steps in the coagulation pathway to provide simpler alternatives to currently available anticoagulants. Among these new agents are direct thrombin inhibitors and direct Factor Xa inhibitors [40]. The direct thrombin inhibitor dabigatran etexilate appears an attractive alternative to the current standard of care in patients after THA and TKA [41-44]. It has been granted marketing authorization in the European Union and Canada for the prevention of VTE after THA or TKA. The manufacturer's recommended dose is $220 \mathrm{mg}$ once daily (starting 1-4 hours after surgery with a single $110 \mathrm{mg}$ capsule) for a total of 28-35 days after THA or a total of 10 days after TKA [45]. Direct Factor Xa inhibitors in development include rivaroxaban, apixaban, edoxaban (DU-176b), and YM150, and of these rivaroxaban is in the most advanced stage of development [46]. Rivaroxaban has shown potential as a once-daily, oral anticoagulant that may be administered in fixed doses for the prevention and treatment of thromboembolic disorders following orthopaedic surgery [47-52]. Rivaroxaban is approved in more than 90 countries worldwide, including the European Union and Canada, for the prevention of VTE after elective hip or knee replacement surgery in adult patients. A dose of $10 \mathrm{mg}$ once daily (with the initial dose 6-10 hours after surgery, provided that haemostasis has been achieved) for 5 weeks after elective hip arthroplasty or 2 weeks after elective knee arthroplasty is recommended by the manufacturer [53].

The main difference between direct thrombin inhibitors and direct Factor Xa inhibitors is their mechanism of action. They also differ in their pharmacokinetic and pharmacodynamic profiles, such as metabolism. For example, in the case of dabigatran, more than $80 \%$ of the systemically available drug is eliminated by renal excretion [54]. Two-thirds of administered rivaroxaban are metabolized to inactive metabolites (half of this is eliminated via the kidneys and half via the fecal route), and one-third is excreted as unchanged active drug in the urine [55].

\section{Conclusion}

The need to use thromboprophylaxis after major orthopaedic surgery is now becoming well recognized. However, adequate administration of thromboprophylaxis regimens does not seem to occur after total joint arthroplasty in a large number of cases. The reasons for this appear complex, involving surgeons' poor awareness of the problem of post-surgical thrombosis, their attitudes to guidelines, concerns about causing bleeding, and the complexities of anticoagulation with current agents. Simplifying therapy, such as once-daily fixed dosing, could change attitudes to anticoagulant use and improve adherence to guidelines. Newly developed, oral, fixed-dose anticoagulants should enable substantial improvement in thromboprophylaxis usage, thereby improving patient outcomes. The primary drawback of the new anticoagulants, particularly those with a long half-life, is the lack of specific antidotes to reverse their anticoagulant effect [56]. Specific antidotes might be needed in particular situations such as for overdose or emergency surgery. However, this may not pertain to dabigatran and rivaroxaban as they have relatively short half-lives (12-14 hours and 7-11 hours, resp.) [45, 53]. As off-label prescribing is not uncommon, there is a risk that new anticoagulants licensed for thromboprophylaxis after THA or TKA will be prescribed for unlicensed indications [57]. These current challenges could be overcome by finding specific antidotes and postapproval surveillance of off-label prescribing.

\section{Acknowledgments}

The author would like to acknowledge Chris Thomas and Li Wan who provided editorial support with funding from Bayer Schering Pharma AG and Johnson \& Johnson Pharmaceutical Research \& Development, L.L.C.

\section{References}

[1] W. H. Geerts, D. Bergqvist, G. F. Pineo et al., "Prevention of venous thromboembolism: American College of Chest Physicians evidence-based clinical practice guidelines (8th edition)," Chest, vol. 133, no. 6 supplement, pp. 381S-453S, 2008.

[2] O. E. Dahl and D. Bergqvist, "Current controversies in deep vein thrombosis prophylaxis after orthopaedic surgery," Current Opinion in Pulmonary Medicine, vol. 8, no. 5, pp. 394-397, 2002.

[3] H. M. Zaw, I. C. Osborne, P. N. Pettit, and A. T. Cohen, "Risk factors for venous thromboembolism in orthopedic surgery," The Israel Medical Association Journal, vol. 4, no. 11, pp. 1040-1042, 2002.

[4] J. A. Caprini and T. M. Hyers, "Compliance with antithrombotic guidelines," Managed Care, vol. 15, no. 9, pp. 49-66, 2006. 
[5] A. K. Kakkar, B. L. Davidson, S. K. Haas et al., "Compliance with recommended prophylaxis for venous thromboembolism: improving the use and rate of uptake of clinical practice guidelines," Journal of Thrombosis and Haemostasis, vol. 2, no. 2, pp. 221-227, 2004.

[6] V. F. Tapson, T. M. Hyers, A. L. Waldo et al., "Antithrombotic therapy practices in US hospitals in an era of practice guidelines," Archives of Internal Medicine, vol. 165, no. 13, pp. 1458-1464, 2005.

[7] D. Warwick, O. E. Dahl, and W. D. Fisher, "Orthopaedic thromboprophylaxis: limitations of current guidelines," Journal of Bone and Joint Surgery. Series B, vol. 90, no. 2, pp. 127-132, 2008.

[8] M. J. Bernstein, H. R. Roberts, and S. Adel, "Prevention of venous thrombosis and pulmonary embolism," Journal of the American Medical Association, vol. 256, no. 6, pp. 744-749, 1986.

[9] J. Ansell, J. Hirsh, E. Hylek, A. Jacobson, M. Crowther, and G. Palareti, "Pharmacology and management of the vitamin K antagonists: American College of Chest Physicians evidence-based clinical practice guidelines (8th Edition)," Chest, vol. 133, no. 6 supplement, pp. 160S-198S, 2008.

[10] J. Hirsh, T. E. Warkentin, S. G. Shaughnessy et al., "Heparin and low-molecular-weight heparin: Mechanisms of action, pharmacokinetics, dosing, monitoring, efficacy, and safety," Chest, vol. 119, no. 1 supplement, pp. 64S-94S, 2001.

[11] Arixtra (fondaparinux sodium)-Prescribing Information, 2005, http://us.gsk.com/products/assets/us_arixtra.pdf.

[12] G. Agnelli, G. B. Mancini, and D. Biagini, "The rationale for long-term prophylaxis of venous thromboembolism," Orthopedics, vol. 23, no. 6 supplement, pp. s643-s646, 2000.

[13] R. J. Friedman, "Optimal duration of prophylaxis for venous thromboembolism following total hip arthroplasty and total knee arthroplasty," Journal of the American Academy of Orthopaedic Surgeons, vol. 15, no. 3, pp. 148-155, 2007.

[14] A. Planes, N. Vochelle, J.-Y. Darmon, M. Fagola, M. Bellaud, and Y. Huet, "Risk of deep-venous thrombosis after hospital discharge in patients having undergone total hip replacement: double-blind randomised comparison of enoxaparin versus placebo," The Lancet, vol. 348, no. 9022, pp. 224-228, 1996.

[15] E. Rahme, K. Dasgupta, M. Burman et al., "Postdischarge thromboprophylaxis and mortality risk after hip- or kneereplacement surgery," Canadian Medical Association Journal, vol. 178, no. 12, pp. 1545-1554, 2008.

[16] R. J. Friedman, A. S. Gallus, F. D. Cushner, G. Fitzgerald, and F. A. Anderson Jr., "Physician compliance with guidelines for deep-vein thrombosis prevention in total hip and knee arthroplasty," Current Medical Research and Opinion, vol. 24, no. 1, pp. 87-97, 2008.

[17] F. A. Anderson Jr., J. Hirsh, K. White, and R. H. Fitzgerald Jr., "Temporal trends in prevention of venous thromboembolism following primary total hip or knee arthroplasty 1996-2001: findings from the hip and knee registry," Chest, vol. 124, supplement 6, pp. 349S-356S, 2003.

[18] D. Warwick, R. J. Friedman, G. Agnelli et al., "Insufficient duration of venous thromboembolism prophylaxis after total hip or knee replacement when compared with the time course of thromboembolic events," Journal of Bone and Joint Surgery. Series B, vol. 89, no. 6, pp. 799-807, 2007.

[19] O. E. Dahl, "Continuing out-of-hospital prophylaxis following major orthopaedic surgery: what now?" Haemostasis, vol. 30, supplement 2, pp. 101-105, 2000.
[20] S. Z. Goldhaber and A. G. Turpie, "Prevention of venous thromboembolism among hospitalized medical patients," Circulation, vol. 111, no. 1, pp. e1-e3, 2005.

[21] C. Kearon, "Duration of venous thromboembolism prophylaxis after surgery," Chest, vol. 124, supplement 6, pp. 386S-392S, 2003.

[22] R. H. White, P. S. Romano, H. Zhou, J. Rodrigo, and W. Bargar, "Incidence and time course of thromboembolic outcomes following total hip or knee arthroplasty," Archives of Internal Medicine, vol. 158, no. 14, pp. 1525-1531, 1998.

[23] S. J. McRae and J. S. Ginsberg, "Initial treatment of venous thromboembolism," Circulation, vol. 110, no. 9, supplement 1, pp. I3-I9, 2004.

[24] P. Prandoni, A. W. A. Lensing, A. Cogo et al., "The long-term clinical course of acute deep venous thrombosis," Annals of Internal Medicine, vol. 125, no. 1, pp. 1-7, 1996.

[25] C. Kearon, "Natural history of venous thromboembolism," Circulation, vol. 107, no. 23, supplement 1, pp. I22-I30, 2003.

[26] S. R. Kahn and J. S. Ginsberg, "Relationship between deep venous thrombosis and the postthrombotic syndrome," Archives of Internal Medicine, vol. 164, no. 1, pp. 17-26, 2004.

[27] V. Pengo, A. W. A. Lensing, M. H. Prins et al., "Incidence of chronic thromboembolic pulmonary hypertension after pulmonary embolism," The New England Journal of Medicine, vol. 350, no. 22, pp. 2257-2323, 2004.

[28] J. A. Caprini, M. F. Botteman, J. M. Stephens et al., "Economic burden of long-term complications of deep vein thrombosis after total hip replacement surgery in the United States," Value in Health, vol. 6, no. 1, pp. 59-74, 2003.

[29] K. K. Knight, J. Wong, O. Hauch, G. Wygant, D. Aguilar, and J. J. Ofman, "Economic and utilization outcomes associated with choice of treatment for venous thromboembolism in hospitalized patients," Value in Health, vol. 8, no. 3, pp. 191-200, 2005.

[30] S. D. Sullivan, S. R. Kahn, B. L. Davidson, L. Borris, P. Bossuyt, and G. Raskob, "Measuring the outcomes and pharmacoeconomic consequences of venous thromboembolism prophylaxis in major orthopaedic surgery," PharmacoEconomics, vol. 21, no. 7, pp. 477-496, 2003.

[31] A. C. Spyropoulos and J. Lin, "Direct medical costs of venous thromboembolism and subsequent hospital readmission rates: an administrative claims analysis from 30 managed care organizations," Journal of Managed Care Pharmacy, vol. 13, no. 6, pp. 475-486, 2007.

[32] B. Detournay, A. Planes, N. Vochelle, and F. Fagnani, "Cost effectiveness of a low-molecular-weight heparin in prolonged prophylaxis against deep vein thrombosis after total hip replacement," PharmacoEconomics, vol. 13, no. 1, pp. 81-89, 1998.

[33] G. Agnelli, M. R. Taliani, and M. Verso, "Building effective prophylaxis of deep vein thrombosis in the outpatient setting," Blood Coagulation and Fibrinolysis, vol. 10, no. 6, supplement 2, pp. S29-S35, 1999.

[34] B. I. Eriksson and D. J. Quinlan, "Oral anticoagulants in development: focus on thromboprophylaxis in patients undergoing orthopaedic surgery," Drugs, vol. 66, no. 11, pp. 1411-1429, 2006.

[35] J. I. Weitz, "Emerging anticoagulants for the treatment of venous thromboembolism," Thrombosis and Haemostasis, vol. 96, no. 3, pp. 274-284, 2006.

[36] M. C. Sokol, K. A. McGuigan, R. R. Verbrugge, and R. S. Epstein, "Impact of medication adherence on hospitalization risk and healthcare cost," Medical Care, vol. 43, no. 6, pp. 521-530, 2005. 
[37] A. Gillissen, "Patients' adherence in asthma," Journal of Physiology and Pharmacology, vol. 58, supplement 5, pp. 205-222, 2007.

[38] B. V. Patel, R. S. Leslie, P. Thiebaud et al., "Adherence with single-pill amlodipine/atorvastatin vs a two-pill regimen," Vascular Health and Risk Management, vol. 4, no. 3, pp. 673-681, 2008.

[39] B. A. Boyle, D. Jayaweera, M. D. Witt, K. Grimm, J.-F. Maa, and D. W. Seekins, "Randomization to once-daily stavudine extended release/lamivudine/efavirenz versus a more frequent regimen improves adherence while maintaining viral suppression," HIV Clinical Trials, vol. 9, no. 3, pp. 164-176, 2008.

[40] J. Ansell, "Factor Xa or thrombin: is factor Xa a better target?" Journal of Thrombosis and Haemostasis, vol. 5, supplement 1, pp. 60-64, 2007.

[41] B. I. Eriksson, O. E. Dahl, N. Rosencher et al., "Oral dabigatran etexilate vs. subcutaneous enoxaparin for the prevention of venous thromboembolism after total knee replacement: the RE-MODEL randomized trial," Journal of Thrombosis and Haemostasis, vol. 5, no. 11, pp. 2178-2185, 2007.

[42] J. S. Ginsberg, B. L. Davidson, P. C. Comp, et al., "Oral thrombin inhibitor dabigatran etexilate vs North American enoxaparin regimen for prevention of venous thromboembolism after knee arthroplasty surgery," Journal of Arthroplasty, vol. 24, no. 1, pp. 1-9, 2009.

[43] B. I. Eriksson, O. E. Dahl, N. Rosencher et al., "Dabigatran etexilate versus enoxaparin for prevention of venous thromboembolism after total hip replacement: a randomised, double-blind, non-inferiority trial," The Lancet, vol. 370, no. 9591, pp. 949-956, 2007.

[44] B. I. Eriksson and R. Friedman, "Dabigatran etexilate: pivotal trials for venous thromboembolism prophylaxis after hip or knee arthroplasty," Clinical and Applied Thrombosis/Hemostasis, vol. 15, pp. 25S-31S, 2009.

[45] Dabigatran Summary of Product Characteristics, "Pradaxa ${ }^{\circledR}$ (dabigatran etexilate) Summary of Product Characteristics," 2008, http://www.pradaxa.com/Include/media/pdf/Pradaxa_ SPC_EMEA.pdf.

[46] K. A. Bauer, "New anticoagulants," Current Opinion in Hematology, vol. 15, no. 5, pp. 509-515, 2008.

[47] B. I. Eriksson, L. C. Borris, O. E. Dahl et al., "A once-daily, oral, direct Factor Xa inhibitor, rivaroxaban (BAY 59-7939), for thromboprophylaxis after total hip replacement," Circulation, vol. 114, no. 22, pp. 2374-2381, 2006.

[48] W. Mueck, B. I. Eriksson, K. A. Bauer et al., "Population pharmacokinetics and pharmacodynamics of rivaroxaban-an oral, direct factor Xa inhibitor-in patients undergoing major orthopaedic surgery," Clinical Pharmacokinetics, vol. 47, no. 3, pp. 203-216, 2008.

[49] B. I. Eriksson, L. C. Borris, R. J. Friedman et al., "Rivaroxaban versus enoxaparin for thromboprophylaxis after hip arthroplasty," The New England Journal of Medicine, vol. 358, no. 26, pp. 2765-2775, 2008.

[50] A. K. Kakkar, B. Brenner, O. E. Dahl et al., "Extended duration rivaroxaban versus short-term enoxaparin for the prevention of venous thromboembolism after total hip arthroplasty: a double-blind, randomised controlled trial," The Lancet, vol. 372, no. 9632, pp. 31-39, 2008.

[51] M. R. Lassen, W. Ageno, L. C. Borris et al., "Rivaroxaban versus enoxaparin for thromboprophylaxis after total knee arthroplasty," The New England Journal of Medicine, vol. 358, no. 26, pp. 2776-2786, 2008.
[52] A. G. G. Turpie, M. R. Lassen, B. L. Davidson et al., "Rivaroxaban versus enoxaparin for thromboprophylaxis after total knee arthroplasty (RECORD4): a randomised trial," The Lancet, vol. 373, no. 9676, pp. 1673-1680, 2009.

[53] Xarelto ${ }^{\circledR}$ Summary of Product Characteristics, 2009, http:// www.xarelto.com/html/downloads/Xarelto_Summary_of_Product_Characteristics_May2009.pdf.

[54] S. Blech, T. Ebner, E. Ludwig-Schwellinger, J. Stangier, and W. Roth, "The metabolism and disposition of the oral direct thrombin inhibitor, dabigatran, in humans," Drug Metabolism and Disposition, vol. 36, no. 2, pp. 386-399, 2008.

[55] C. Weinz, T. Schwarz, D. Kubitza, W. Mueck, and D. Lang, "Metabolism and excretion of rivaroxaban, an oral, direct factor xa inhibitor, in rats, dogs, and humans," Drug Metabolism and Disposition, vol. 37, no. 5, pp. 1056-1064, 2009.

[56] J. I. Weitz, J. Hirsh, and M. M. Samama, "New antithrombotic drugs: American College of Chest Physicians evidence-based clinical practice guidelines (8th edition)," Chest, vol. 133, no. 6 supplement, pp. 234S-256S, 2008.

[57] D. C. Radley, S. N. Finkelstein, and R. S. Stafford, "Offlabel prescribing among office-based physicians," Archives of Internal Medicine, vol. 166, no. 9, pp. 1021-1026, 2006. 


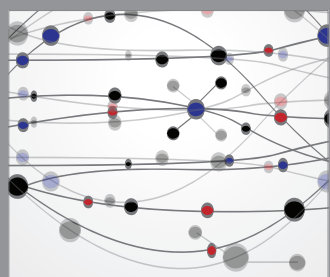

The Scientific World Journal
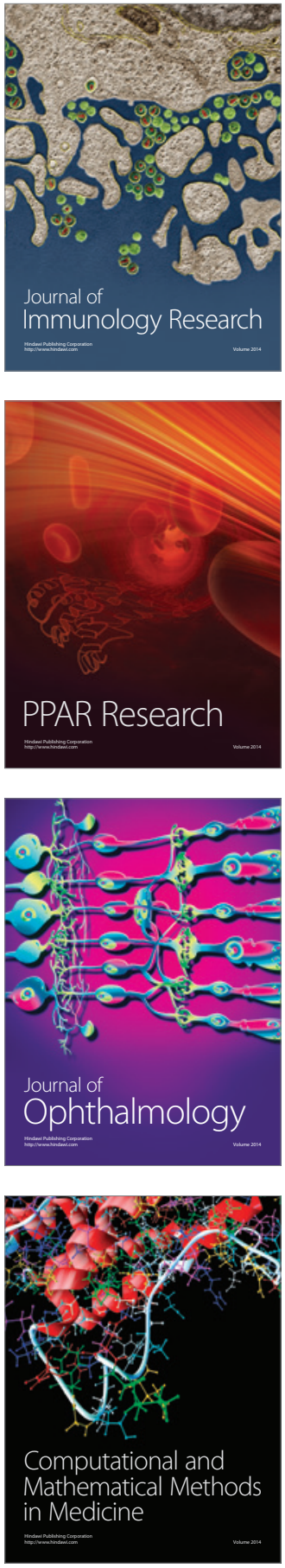

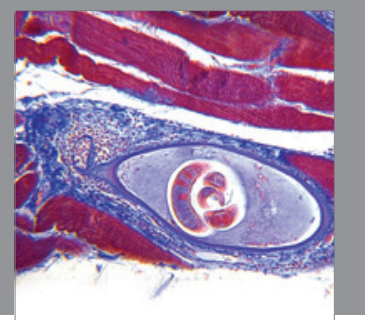

Gastroenterology

Research and Practice
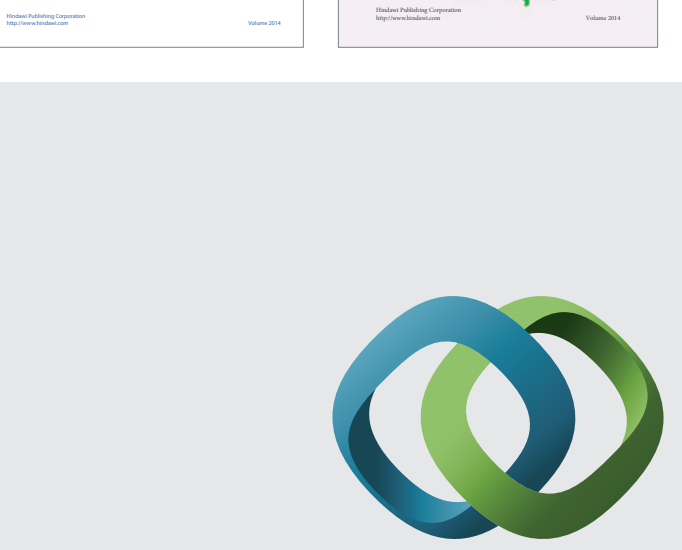

\section{Hindawi}

Submit your manuscripts at

http://www.hindawi.com
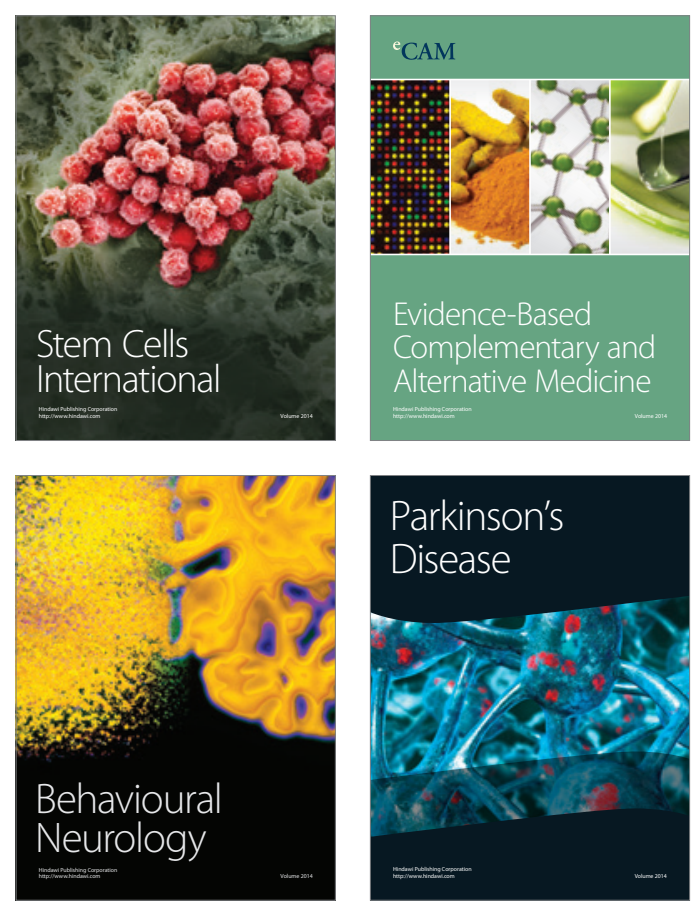

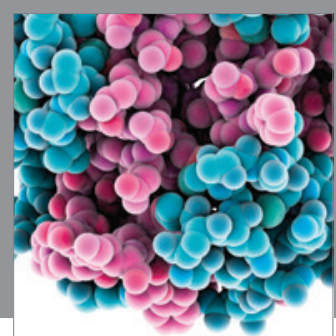

Journal of
Diabetes Research

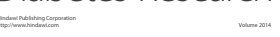

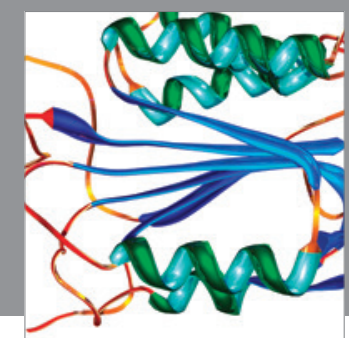

Disease Markers
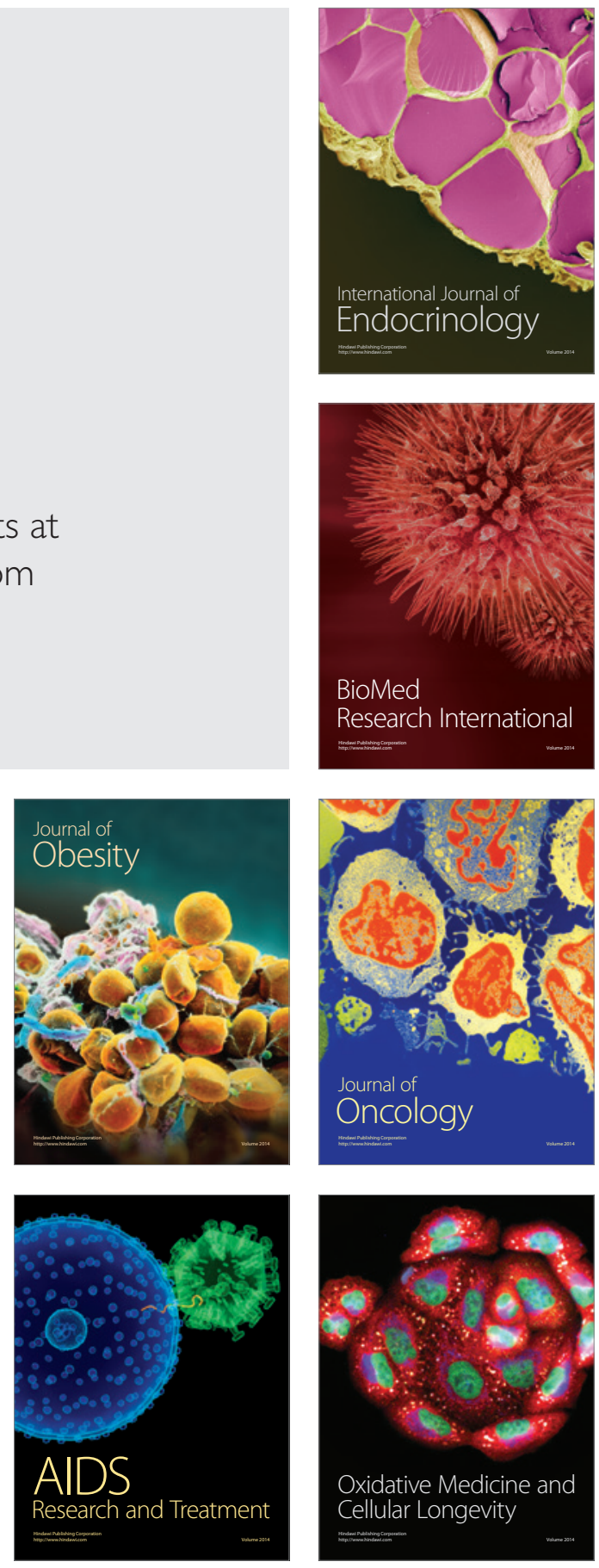